\section{Identificação do limiar anaeróbio ventilatório em crianças e adolescentes: revisão da literatura}

\section{Identifying ventilatory anaerobic threshold in children and adolescents: A literature review}

Anselmo José Perez ${ }^{1}$

Luciana Carletti

Resumo - O limiar anaeróbio ventilatório (LAV) tem sido utilizado em pesquisas para representar a função pulmonar e a capacidade de desempenho submáximo de crianças e adolescentes. O estudo teve como objetivo identificar, a) a que grupo de crianças e adolescentes tem sido o principal foco dos trabalhos; b) quais critérios têm sido utilizados para identificação do LAV; c) quais são as principais referências bibliográficas utilizadas e em que se apóiam as suas fundamentações teóricas. Foi feito um levantamento de trabalhos que tenham utilizado o LAV como metodologia de estudo nas bases de dados LILACS, MEDLINE, SCIELO, e PUBMED no período de 2000 a 2010 para identificação de categorias por meio de análise de conteúdo. Apresentação dos dados pela estatística descritiva. Foram encontrados quarenta e um trabalhos, que se distribuíram assim, de acordo com a categoria identificada: intolerância severa ao exercício $(21=51 \%)$; saudáveis $(6=15 \%)$; obesas e comparação de metodologias ( $4=10 \%$, cada); cinética de $\mathrm{O}_{2}(3=7 \%)$; esporte $(2=5 \%)$; desnutrição pregressa, asma, e percepção do esforço $(1=2 \%$, cada). O principal critério tem sido o V-slope e a principal referência utilizada é Beaver WL, Wasserman K, Whipp BJ (1986), com 24 ocorrências (53\%). Além desse método, os equivalentes ventilatórios de oxigênio e de dióxido de carbono tem sido utilizados, o que representou $37 \%$ (17 trabalhos). Conclusão: os dados indicam que a principal utilização do LAV tem sido para estudos de reabilitação de crianças e adolescentes por meio da técnica do V-slope.

Palavras-chave: Adolescentes; Consumo de oxigênio; Criança; Limiar anaeróbio; Metabolismo.

Abstract - Ventilatory anaerobic threshold (VAT) has been used in research to represent pulmonary function and submaximal performance capacity of children and adolescents. This study aimed to identify: a) the group of children and adolescents that has been the main focus of research; $b$ ) the criteria most commonly used to determine VAT; and c) the main references that have been used to support the theoretical analysis. A literature search was conducted using LILACS, MEDLINE/PubMed, and SciELO. The search was limited to studies using VAT in their methodology, published between 2000 and 2010, in order to identify categories through content analysis. Descriptive statistics were used to analyze the data. Forty-one studies were found and distributed according to categories identified, as follows: severe exercise intolerance (21 [51\%]); healthy subjects (6 [15\%]); obese subjects and comparison of methodologies (4 [10\%], each); $\mathrm{O}_{2}$ kinetics (3 [7\%]); sports (2 [5\%]); stunting, asthma, and effort perception (1 [2\%], each). The main reference used is Beaver WL, Wasserman K, Whipp BJ (1986), cited in 24 (53\%) studies, and the main criterion for $V A T$ determination is the $V$-slope method. In addition to this method, ventilatory equivalents for $\mathrm{O}_{2}$ and $\mathrm{CO}_{2}$ have been used, accounting for 37\% (17) of cases. In conclusion, the data indicate that VAT has been primarily used in rehabilitation studies including children and adolescents by the $V$-slope method.

Key words: Adolescent; Anaerobic threshold; Child; Metabolism; Oxygen consumption.
1 Universidade Federal do Espírito Santo. Espírito Santo, ES. Brasil

Recebido em 18/04/10 Revisado em 31/10/11 Aprovado em 21/12/11

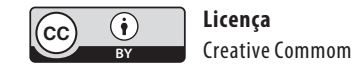




\section{INTRODUÇÃO}

Os critérios para o exercício máximo descritos em adultos são raramente satisfeitos em crianças e adolescentes ${ }^{1}$ e, portanto, necessitam de maior especificidade e precisão. Variáveis cardiorrespiratórias, como ventilação pulmonar (VE), razão das trocas respiratórias (RTR), frequência respiratória, pulso de oxigênio, e frequência cardíaca (FC), medidas durante os testes físicos de exercício progressivo, têm valores máximos nas crianças e adolescentes muito diferentes dos valores apresentados por adultos ${ }^{2}$.

O consumo máximo de oxigênio $\left(\mathrm{VO}_{2 \text { max }}\right)$ e/ou pico de consumo de oxigênio $\left(\mathrm{VO}_{2 \text { pico }}\right)$, assim como o $\mathrm{VO}_{2}$ no limiar anaeróbio ventilatório (LAV), são os parâmetros utilizados para a avaliação de aptidão aeróbia de crianças e adolescentes ${ }^{3}$, para níveis máximos e submáximos, respectivamente. Além disso, a eficiência ventilatória, examinada em determinados pontos do exercício, por meio do LAV, ou expressa como a inclinação da relação entre a VE e a produção de dióxido de carbono $\left(\mathrm{VCO}_{2}\right)$, tem sido proposta como importante indicador de saúde para a faixa de idade em questão ${ }^{4}$. Os valores elevados deste parâmetro indicam ventilação ineficiente e estão presentes no perfil funcional de uma série de doenças cardíacas e pulmonares ${ }^{5}$ e têm sido estudados em crianças saudáveis para a identificação de parâmetros de referência para o processo de crescimento e desenvolvimento ${ }^{6-8}$

Durante os testes de exercício incremental, Wasserman e Mcllroy ${ }^{9}$ notaram que existia certo ponto em que a VE aumentava além da proporção do consumo de oxigênio $\left(\mathrm{VO}_{2}\right)$. Na época, este ponto foi denominado LAV, indicando o aparecimento de metabolismo anaeróbio (limiar anaeróbio) e, como houve mensuração invasiva da concentração do lactato sanguíneo caracterizando o ponto no qual este passava a se acumular acima do nível de repouso durante o exercício incremental ${ }^{10}$, foi postulada a ligação entre o primeiro aumento na concentração de lactato sanguíneo e algumas alterações previsíveis dos parâmetros de troca gasosa, permitindo inferir que o limiar anaeróbio (lactato) pode ser determinado com precisão pela resposta ventilatória ${ }^{10-12}$, apesar de controvérsias a esse fato ${ }^{12-14}$. $\mathrm{O}$ conceito inicial de LAV proposto por Wasserman e colaboradores ${ }^{9,10,12}$ ainda parece prevalecer quando o tema é índice de resistência física e identificação da intensidade do exercício em programas de treinamento físico.

Desde então, diversos indicadores têm sido sugeridos para a determinação do LAV, como relação entre $\mathrm{VCO}_{2}$ e $\mathrm{VO}_{2}$, o equivalente ventilatório de oxigênio $\left(\mathrm{VE} / \mathrm{VO}_{2}\right)$, o equivalente ventilatório de dióxido de carbono (VE/ $\mathrm{VCO}_{2}$ ), ou a razão de troca respiratória (RTR), analisados ao longo do tempo de exercício ou do $\mathrm{VO}_{2}^{15,16}$. A combinação de vários parâmetros parece aumentar a confiabilidade da identificação do LAV em crianças e adolescentes ${ }^{3,17,18}$.

Considerando que estudos que esclareçam sobre a fisiologia cardiorrespiratória de crianças e adolescentes são necessários, por revelarem condições clínicas e de aptidão, nesta população diferenciada; e ainda que especialmente o LAV vem sendo identificado a partir de critérios diversos; elaborou-se essa revisão para responder as seguintes questões: a) qual 
grupo de crianças e adolescentes tem sido o principal foco das pesquisas que utilizaram o LAV como variável de estudo; b) quais critérios têm sido utilizados para identificação do LAV; c) quais são as principais referências bibliográficas utilizadas e em que se apóiam as suas fundamentações teóricas.

Trata-se de uma revisão da literatura nas bases de dados LILACS, MEDLINE, SCIELO, e PUBMED dos últimos 10 anos, utilizando os seguintes descritores: criança, adolescentes, e limiar anaeróbio. Para seleção dos trabalhos considerou-se a apresentação da identificação do LAV na metodologia e, excluiu-se aqueles que utilizavam apenas a mensuração do lactato como metodologia de identificação do limiar anaeróbio, uma vez que, não havia interesse em se estudar limiar de lactato. Os trabalhos foram catalogados, analisados e procurou-se identificar os principais focos de pesquisa, apresentando-se sugestão de categorias a partir da análise de conteúdo relacionado à amostra estudada.

\section{IDENTIFICAÇÃO DOS TRABALHOS}

Quarenta e um trabalhos puderam ser analisados, e de acordo com a população de crianças e adolescentes focalizadas foram distribuídos com as seguintes categorias: aquelas que apresentavam quadro clínico associado à intolerância severa ao exercício físico, como insuficiência cardíaca congênita e reparo cirúrgico, miosite inflamatória, leucemia, operação de Fontain, lúpus pediátrico, doença renal, paralisia cerebral, doença pulmonar crônica, ou escoliose $(21=51 \%)$; saudáveis $(6=15 \%)$; aquelas que passaram por diferentes protocolos para comparação de metodologias para testes cardiopulmonares ( $4=10 \%)$; aquelas que realizaram teste para o estudo da cinética de $\mathrm{O}_{2}(3=7 \%)$; obesas $(2=5 \%)$; que participaram de programas de treinamento de esportes $(2=5 \%$, cada categoria); e que apresentavam desnutrição pregressa, ou asma, ou que tiveram a percepção subjetiva de esforço estudada ( $1=2 \%$, cada categoria).

No Quadro 1 encontra-se, de acordo com as categorias criadas, a identificação em ordem cronológica decrescente dos autores dos trabalhos com as variáveis: idade, gênero e o número (n) da amostra de crianças e adolescentes, tipo de ergômetro utilizado no protocolo de teste cardiopulmonar, se foi usado máscara ou bocal com clipe nasal, e o critério de identificação do LAV.

Pode-se observar que o principal critério adotado para identificação do LAV foi o V-slope $(18=40 \%)$ utilizando preferencialmente a referência de Beaver et al. ${ }^{15}$, com 25 ocorrências (56\%). Outro critério utilizado por $20 \%$ dos trabalhos $(\mathrm{n}=9)$ foi dos equivalentes ventilatórios de oxigênio e de dióxido de carbono. Alguns trabalhos descreveram a utilização de mais de um identificador para o LAV. Assim, cinco (11\%) utilizaram ambos V-slope e equivalente ventilatório, quatro (9\%) utilizaram equivalente ventilatório e as medidas das pressões expiradas de oxigênio $\left(\mathrm{P}_{\mathrm{et}} \mathrm{O}_{2}\right)$ e de dióxido de carbono $\mathrm{P}_{\mathrm{et}} \mathrm{CO}_{2}$. Dois descreveram os três critérios já citados, um incluiu a RTR em substituição ao $\mathrm{P}_{\text {et }} \mathrm{O}_{2}$, um trabalho utilizou o equivalente e a RTR, e um citou Wasserman et al. ${ }^{10}$, subentendendo que a quebra da linearidade da ventilação e do $\mathrm{CO}_{2}$, assim como a $\mathrm{P}_{\mathrm{et}} \mathrm{O}_{2}$ foram utilizadas. 
Quadro 1. Estudos de acordo com a categoria envolvendo crianças e adolescentes.

\begin{tabular}{|c|c|c|c|c|c|c|}
\hline & Autores & Idade & Gênero/n & $\begin{array}{c}\text { Tipo } \\
\text { Ergômetro }\end{array}$ & $\begin{array}{c}\text { Máscara/ } \\
\text { Bocal }\end{array}$ & Identificação LAV \\
\hline \multirow{21}{*}{$\begin{array}{l}\text { Intolerância } \\
\text { exercício }\end{array}$} & Möller P et al 2009 & $4-11$ & Ambos/45 & Esteira & M & V-slope \\
\hline & Amiard V et al 2008 & $13-16$ & Ambos/35 & Bicicleta & B & V-slope \\
\hline & Houghton KM et al 2008 & $12-18$ & Ambos/15 & Esteira & B & Equivalentes ventilatórios \\
\hline & Meadows J et al 2008 & $5-46$ & Indis/20 & Bicicleta & Indis & V-slope \\
\hline & Paridon S M et al 2008 & $8-15$ & Ambos/411 & Bicicleta & Indis & V-slope \\
\hline & San Juan AF et al 2008 & $4-10$ & Ambos/15 & Esteira & M & Equivalentes ventilatórios e $\mathrm{P}_{\mathrm{et}} \mathrm{O}_{2}$ \\
\hline & Sritippayawan S et al 2008 & $11-16$ & Ambos/36 & Bicicleta & M & $\begin{array}{c}\text { V-slope ou equivalentes ventila- } \\
\text { tórios ou RTR }>1,0\end{array}$ \\
\hline & Takken T et al 2008 & $8-13$ & Ambos/13 & Bicicleta & M & Equivalentes ventilatórios e $\mathrm{P}_{\mathrm{et}} \mathrm{O}_{2}$ \\
\hline & Amiard V et al 2007 & $10-16$ & Ambos/35 & Bicicleta & $\mathrm{B}$ & V-slope \\
\hline & Hager A et al 2007 & $8-18$ & Ambos/55 & Bicicleta & Indis & V-slope e equivalentes ventilatórios \\
\hline & McBride MG et al 2007 & $7-15$ & Ambos/27 & Bicicleta & Indis & V-slope \\
\hline & McCrindle BW et al 2007 & $6-18$ & Ambos/103 & Bicicleta & Indis & V-slope \\
\hline & Yasuda K et al 2007 & $5-13$ & Ambos/19 & Esteira & $\mathrm{M}$ & *Ventilação e $\mathrm{P}_{\mathrm{et}} \mathrm{O}_{2}$ \\
\hline & Davis JA et al 2006 & $10-15$ & Ambos/28 & Bic ou estei & Indis & V-slope \\
\hline & Moalla W et al 2005 & $12-16$ & 31 & Bicicleta & $\mathrm{B}$ & V-slope \\
\hline & Rhodes J et al 2005 & $8-17$ & Ambos/16 & Bicicleta & Indis & V-slope \\
\hline & Pattaragarn A et al 2004 & $11-18$ & Ambos/32 & Esteira & Indis & Equivalentes ventilatórios \\
\hline & Shinohara T et al 2002-2003 & $12-16$ & 11 & $\begin{array}{c}\text { Bicicleta } \\
\text { ergo braço }\end{array}$ & M & V-slope \\
\hline & Guimarães GV et al 2001 & $8-10$ & Ambos/38 & Esteira & B & Equivalentes ventilatórios e $\mathrm{P}_{\mathrm{et}} \mathrm{O}_{2}$ \\
\hline & Sato T et al 2001 & 8-19 & 70 & Bicicleta & Indis & $\begin{array}{l}\text { V-slope e equivalentes ventilató- } \\
\text { rios e } \mathrm{P}_{\mathrm{et}} \mathrm{O}_{2}\end{array}$ \\
\hline & Reybrouck T et al 2000 & $10-12$ & Ambos/81 & Esteira & Indis & V slope \\
\hline \multirow{6}{*}{ Saudáveis } & Prado DML et al 2010 & $\begin{array}{c}10-11 /- \\
28\end{array}$ & Ambos/45 & Esteira & Indis & $\begin{array}{l}\text { V-slope ou equivalentes ventila- } \\
\text { tórios }\end{array}$ \\
\hline & Guerrero, L et al 2008 & $7-9$ & Ambos/84 & Bicicleta & $M$ & Equivalentes ventilatórios \\
\hline & Chintala K et al 2008 & $8-18$ & Ambos/24 & Esteira & B & Equivalentes ventilatórios \\
\hline & Mandadzhieva S et al 2005 & $10-11$ & Ambos/153 & Esteira & Indis & V-slope e equivalentes ventilatórios \\
\hline & Gürsel Y et al 2004 & $5-13$ & Ambos $/ 90$ & Esteira & Indis & Equivalentes ventilatórios \\
\hline & Al-Hazzaa H 2001 & $7-15$ & Masc/137 & Esteira & Indis & Equivalentes ventilatórios \\
\hline \multirow{4}{*}{$\begin{array}{l}\text { Compara- } \\
\text { ção meto- } \\
\text { dologias }\end{array}$} & Dubowy Karl-Otto et al 2008 & $4-75$ & Ambos/1195 & Esteira & Indis & V-slope \\
\hline & Anderson CS; Mahon A 2007 & $\begin{array}{c}10- \\
12 / 21-27\end{array}$ & Masc/17 & Bicicleta & B & Equivalentes ventilatórios \\
\hline & Moalla W et al 2005 & $12-14$ & Masc/12 & Bicicleta & B & V-slope \\
\hline & Hebestreit, H et al 2000 & $6-12$ & Ambos/55 & Bicicleta & B & Equivalentes ventilatórios e RTR \\
\hline \multirow{3}{*}{ Cinética $\mathrm{O}_{2}$} & $\begin{array}{l}\text { Fawkner SGF, Armstrong } \\
\text { N } 2004\end{array}$ & $10-11$ & Ambos/22 & Bicicleta & Indis & V-slope \\
\hline & $\begin{array}{l}\text { Fawkner SG; Armstrong N } \\
\text { 2004b }\end{array}$ & $10-11$ & Ambos/48 & Bicicleta & Indis & V-slope \\
\hline & Fawkner SG et al 2002 & $11-12$ & Ambos/23 & Bicicleta & Indis & Equivalentes ventilatórios e $\mathrm{P}_{\mathrm{et}} \mathrm{O}_{2}$ \\
\hline \multirow{2}{*}{ Esporte } & Mikulić P; Ruzić L 2008 & $12-14$ & Masc/48 & Esteira & Indis & V-slope \\
\hline & Debray P; Swapan KD 2007 & $11-13$ & Masc/26 & Esteira & B & Equivalentes ventilatórios \\
\hline \multirow{2}{*}{ Obesas } & Prado DM et al 2009 & $8-12$ & 48 & Esteira & B & $\begin{array}{l}\text { V-slope ou equivalentes ventila- } \\
\text { tórios e } \mathrm{P}_{\mathrm{et}} \mathrm{O}_{2}\end{array}$ \\
\hline & Marinov B et al 2002 & $7-14$ & Ambos/60 & Esteira & B & $\begin{array}{l}\begin{array}{l}\text { V-slope ou equivalentes ventila- } \\
\text { tórios }\end{array}\end{array}$ \\
\hline $\begin{array}{l}\text { Desnutrição } \\
\text { pregressa }\end{array}$ & Fernandez AC et al 2000 & $8-11$ & Fem/24 & Bicicleta & Indis & Equivalentes ventilatórios e $R T R \geq 1,0$ \\
\hline Asma & Counil FP et al 2003 & $10-16$ & Masc/14 & Bicicleta & Indis & V-slope \\
\hline $\begin{array}{l}\text { Percepção } \\
\text { subjetiva }\end{array}$ & Robertson RJ et al 2001 & $8-12$ & Ambos/ 48 & Bicicleta & B & Equivalentes ventilatórios \\
\hline
\end{tabular}

Legenda e detalhes: Máscara (M); Bocal (B). Identificação LAV = V-Slope (relação $\mathrm{VCO}_{2}$ e V0) ventilatórios ( $\uparrow V E / V O$ sem $\uparrow V E / V C O_{2}$, e em alguns casos também $\uparrow P_{\text {et }} 0_{2}$ ). ${ }^{*} 0$ s autores citam critério utilizado por Wasserman $\mathrm{K}$ et al. [10], que por sua vez se referem a quebra da linearidade da VE e $\mathrm{P}_{\text {et }} \mathrm{O}_{2}$ como parâmetros a serem utilizados. 
Em relação aos ergômetros, a maioria utilizou a bicicleta eletromagnética (24=59\%); 17 (41\%) utilizaram a esteira e dois não identificaram.

Menos apresentado na metodologia foi o instrumento para coleta do ar durante o teste, já que dos 41 trabalhos, 21 (51\%) não disponibilizaram na descrição se foi utilizada a máscara ou o bocal, com clipe nasal. Dos que apresentaram, 13 (65\%) era por meio de bocal, e apenas 7 (35\%) máscaras.

\section{FOCO DAS PESQUISAS}

Apesar de subjetivas e sem a preocupação de utilização e caracterização de palavras chave, as categorias levantadas permitem identificar os rumos seguidos pelos pesquisadores na última década. Assim, os dados mostram que a aplicação clínica é a mais visada, tanto para o conhecimento efetivo das limitações apresentadas pelas crianças e adolescentes como para o acompanhamento de efeitos do treinamento físico e os seus benefícios para tais limitações.

Para nossa surpresa, esse panorama não apresenta equilíbrio entre interesses por estudos com indivíduos normais e praticantes de esportes e atletas, principalmente adolescentes. Esperávamos que adolescentes atletas fossem alvo de um maior número de pesquisas que enfocassem os efeitos do treinamento físico em diversas modalidades esportivas, já que representam a base de formação esportiva profissional em todo mundo. Além disso, no Brasil nos encontramos na expectativa de sediarmos o evento esportivo mais importante do mundo em 2016, que são os Jogos Olímpicos, e a formação de jovens atletas também se faz por meio de pesquisas das condições cardiorrespiratórias e metabólicas.

Portanto, desde o final do século passado o teste de esforço é empregado como método avaliativo da aptidão física aeróbia de pacientes na pediatria, envolvendo crianças e adolescentes com condições crônicas de saúde ${ }^{17}$. Essa tendência continua nessa primeira década do século XXI, como pode ser visto no Quadro 1.

\section{LAV FOI 0 MÉTODO/TÉCNICA MAIS UTILIZADA}

Não fica dúvida sobre o fato de o LAV fornecer um bom marcador para o condicionamento aeróbio ${ }^{17,19-22}$, e por isso vem sendo usado com frequência desde a década de 80 em crianças e adolescentes saudáveis ${ }^{3,20,23}$, com condição crônica de doença ${ }^{20}$, como também como acompanhamento dos efeitos do treinamento de resistência ${ }^{19}$. O LAV é considerado um marcador confiável de aptidão aeróbia devido à sua capacidade de ser sustentado por um longo período de tempo e de sua sensibilidade ao descondicionamento aeróbio e sedentarismo ${ }^{24}$. Além disso, ele melhora após treinamento esportivo em crianças saudáveis ${ }^{17}$ e com intolerância severa ao exercício ${ }^{25}$, tornando-se então um bom parâmetro para avaliação de programas de treinamento físico. Rhodes et al. ${ }^{26}$ aplicaram o LAV em programa de rea- 
bilitação e identificaram que a sua variação (delta) era um importante fator para identificação do efeito do programa em crianças.

A fundamentação teórica apresentada por Wasserman et al. ${ }^{10}$, a qual mostra a relação entre a oferta de oxigênio e a produção de ácido lático, apresentadas inicialmente com o conceito de Hill-Meyerhof de inadequação no transporte de $\mathrm{O}_{2}$, ainda parece prevalecer. Isso foi apresentado em vários outros trabalhos ${ }^{12,27-29}$.

A vantagem do LAV é que ele pode ser determinado de forma não invasiva durante o exercício sem a necessidade de um esforço máximo ${ }^{30}$, dependendo menos do fator motivação da criança durante o teste ${ }^{31}$. Porém, Moalla et al. ${ }^{32}$ lembram que quando se pretende relacionar o LAV com o $\mathrm{VO}_{2 \text { máx }}$ ou $\mathrm{VO}_{2 \text { pico }}$ é necessário ficar atento a esse fator, já que algumas crianças podem ter os seus resultados descartados.

Segundo Beaver et al. ${ }^{15}$ a verificação de uma transição na relação entre $\mathrm{VCO}_{2}$ e $\mathrm{VO}_{2}$ é um elemento fundamental em todas as técnicas para detectar o LAV por troca de gases. Em um teste de exercício com carga progressiva essa transição pode representar o aumento progressivo e exponencial na produção de lactato (limiar de lactato), em consequência, acumula-se lactato, gerando acidose metabólica e hiperventilação. Com isso, a capacidade de exercício começa a ser prejudicada ${ }^{12,14,30}$, ocorrendo nessa fase o tamponamento de ácido lático pelo bicarbonato com conseqüente aumento do $\mathrm{VCO}_{2}$.

Como confirmado nesta revisão, de todos os métodos de determinação do LAV, o mais utilizado é o V-slope, como citado por Moalla et al. ${ }^{32}$. Verifica-se que essa técnica baseia-se na determinação do ponto de aumento não-linear da inclinação da produção de dióxido de carbono $\left(\mathrm{VCO}_{2}\right)$ versus consumo de oxigênio $\left(\mathrm{VO}_{2}\right)$ durante teste de esforço por meio de exercício incremental. Esse método pode ser facilmente empregado pela plotagem das variáveis $\mathrm{VCO}_{2} \mathrm{e} \mathrm{VO}_{2}$, respectivamente, no eixo da ordenada e abscissa de um gráfico, e observada visualmente por avaliadores experientes sem prejuízo na determinação do LAV, mesmo considerando a subjetividade do método ${ }^{32}$. Para identificação do LAV a referência mais citada foi a de Beaver et al. ${ }^{15}$, com $65 \%$ de ocorrências. Neste trabalho supracitado, o grupo de Karl Wasserman apresenta a proposta de V-slope por meio de método computadorizado, após comparação com a técnica visual de identificação do ponto de limiar anaeróbio seguida na época naquele laboratório. Após registrar $\mathrm{VE} / \mathrm{VCO}_{2}, \mathrm{VE} / \mathrm{VO}_{2}, \mathrm{P}_{\mathrm{et}} \mathrm{CO}_{2}, \mathrm{P}_{\mathrm{et}} \mathrm{O}_{2} \mathrm{e}$ RTR vs tempo, seguindo: 1) a curva $\mathrm{VE} / \mathrm{VO}_{2}$ está plana (na horizontal) ou diminuindo, e começa a aumentar à medida que a curva de $\mathrm{VE} / \mathrm{VCO}_{2}$ permanece constante ou diminui; 2) A curva $\mathrm{P}_{\text {et }} \mathrm{CO}_{2}$-trabalho está subindo lentamente ou está constante, mas a curva $\mathrm{P}_{\mathrm{et}} \mathrm{O}_{2}$-trabalho, está diminuindo ou na horizontal, e começa a subir; 3) A curva de RTR-trabalho está na horizontal ou lentamente subindo, com mudanças para uma inclinação positiva; seis experientes avaliadores compararam os resultados. Como esse método está disponível em sistemas comerciais de teste cardiopulmonar a sua praticidade, além de outros fatores, parece facilitar a sua aplicação, 
principalmente em laboratórios e clínicas de programas de reabilitação. $\mathrm{Na}$ proposta do $\mathrm{V}$-slope, o $\mathrm{VO}_{2}$ é usado como variável independente, porque é o índice direto do metabolismo, baseando-se na análise das inclinações das curvas de volume de $\mathrm{O}_{2}$ e $\mathrm{CO}_{2}{ }^{15}$.

\section{OS OUTROS CRITÉRIOS}

Além da utilização do V-slope para determinação do LAV, os equivalentes ventilatórios de oxigênio $\left(\mathrm{VE} / \mathrm{VO}_{2}\right)$ e de dióxido de carbono $\left(\mathrm{VE} / \mathrm{VCO}_{2}\right)$, e RTR tem sido utilizados. Menos de 20\% dos trabalhos utilizaram somente os equivalentes respiratórios. Mas, esse critério parece ser um importante aliado à interpretação do LAV quando se utiliza o V-slope. Por sua vez, a RTR foi citada em três dos trabalhos estudados. Fernandez et al. ${ }^{33}$ que utilizaram Zeballos e Weisman ${ }^{34}$ como referência, e Sritippayawan et al. ${ }^{35}$ que citam Marinov et al. ${ }^{16}$ e Solberg et al. ${ }^{36}$, descrevem a necessidade de identificação da RTR maior ou igual a 1,0, enquanto Hebestreit, et al. ${ }^{17}$ não caracterizam dimensão, mas sim a sua interpretação em relação ao tempo, sugerindo a identificação da quebra de linearidade dessa variável.

Apesar de não terem sido citados em nenhum desses três trabalhos, Solberg et al. ${ }^{36}$ concluíram que a RTR $>1,0$ é um bom parâmetro para identificação da LAV em adultos, apesar de revelar valores mais elevados do que o método de V-slope. Marinov et al. ${ }^{16}$ observaram RTR maior para crianças obesas, considerando a dependência de uma atividade anaeróbia maior.

É importante observar que a $\mathrm{P}_{\text {et }} \mathrm{O}_{2}$ é citada na metodologia de sete trabalhos. Assim, a sugestão apresentada por alguns autores parece ser considerada sobre combinação de vários parâmetros para aumentar a confiabilidade da identificação do LAV em crianças e adolescentes ${ }^{3,17,18}$. Moalla et al. ${ }^{32}$ sugerem a técnica da espectroscopia no infravermelho próximo (near infrared spectroscopy - NIRS) para análise da desoxigenação dos músculos respiratórios e identificação do limiar anaeróbio, já que se assemelha a técnica do V-slope.

\section{CONDIÇÕES METODOLÓGICAS DE APLICAÇÃO DO MÉTODO}

Uma série de estudos tem indicado que o LAV ocorre geralmente em aproximadamente $60 \%-70 \%$ do $\mathrm{VO}_{2 \text { máx }}{ }^{17,20}$. A consistência entre os estudos pode ser observada independente das variações no modo de exercício, do incremento em fase de teste e duração, dos parâmetros ventilatórios utilizados para determinar o LAV, ou do sistema de análise de gás e processos de amostragem ${ }^{13}$. Identifica-se também que a técnica de respiração por respiração por meio de análise de gases tem sido considerada adequada para a avaliação e interpretação dos dados da cinética do $\mathrm{VO}_{2}$ proveniente dos estudos de exercícios em crianças ${ }^{37}$.

Com relação ao ergômetro, a maioria utilizou a bicicleta eletromagnética. Na comparação entre os dois ergômetros, Wasserman et al. ${ }^{38}$ dão preferência à bicicleta na avaliação clínica, apesar de citarem que a maioria 
dos indivíduos tem pico de $\mathrm{VO}_{2}$ e LA mais baixos na bicicleta ergométrica, sendo os valores máximos de FC, VE e lactato sanguíneo similares. Assim, a escolha do ergômetro entre bicicleta e esteira pode ser uma questão de preferência ou cultura/tradição dos laboratórios e seus pesquisadores em suas regiões.

Em relação à utilização de máscara ou bocal com clipes no nariz, apesar de não ter sido apresentada pela maioria dos trabalhos, observamos que para aqueles que descreveram, o maior percentual de escolha de bocal ao invés de máscara pode estar associado a atual disposição desta última nos laboratórios. Wasserman et al. ${ }^{38}$ apontam para o conforto da máscara e relatam, no entanto, que o espaço morto é em geral ligeiramente maior com as máscaras do que com os bocais.

\section{CONSIDERAÇÕES FINAIS}

Nosso estudo se justifica pela necessidade de se desenvolver investigações de valores de referência de limiar anaeróbio em crianças e adolescentes, assim como os mecanismos fisiológicos envolvidos com grupos nessas faixas etárias, e isso envolve os métodos utilizados. Como o método não invasivo por meio de teste cardiopulmonar atende às exigências metodológicas e éticas, é fundamental para efeitos comparativos tomada de decisão na escolha dos parâmetros a serrem analisados.

Os dados dessa revisão nos remetem para o seguinte: a) o grupo para o qual se tem dado principal foco para as pesquisas que utilizaram o LAV como variável de estudo, continua sendo de crianças e adolescentes com intolerância severa ao exercício físico. No entanto, ainda não se tem dados normalizados para crianças saudáveis; b) dos critérios sugeridos na literatura, o registro gráfico da relação $\mathrm{VCO}_{2} \mathrm{com} \mathrm{o} \mathrm{VO}_{2}$ em teste de carga progressiva por meio do método V-slope têm sido o mais utilizado para identificação do LAV; c) a fundamentação teórica e resultados práticos de pesquisas apresentadas por Wasserman e seus colaboradores sustentam os critérios e o método de V-slope para prática da identificação do limiar anaeróbio em crianças e adolescentes; porém, a correção do termo LAV para LA deve ser feita.

Como sugestão, apresentamos a necessidade de trabalhos voltados a grupos de praticantes e não praticantes de atividades corporais regulares, grupos específicos de atletas em formação, bem como estudos voltados a identificação de valores de referência para crianças e adolescentes saudáveis.

\section{REFERÊNCIAS BIBLIOGRÁFICAS}

1. Karila C, de Blic J, Waernessyckle S, Waernessyckle S, Benoist M-R, Scheinmann P. Cardiopulmonary exercise testing in children: an individualized protocol for workload increase. Chest 2001;120;81-7.

2. Armstrong N, Welsman J, Winsley R. Is peak VO2 a maximal index of children's aerobic fitness? Int J Sports Med 1996;17(5):356-9.

3. Washington RL. Anaerobic threshold in children. Ped Exer Sci 1989;1:244-56.

4. Guerrero L, Naranjo J, Carranza M. Influence of gender on ventilatory efficiency 
during exercise in young children. J Sports Sci 2008;26(13):1455-7.

5. Habedank D, Reindl I, Vietzke G, Bauer U, Sperfeld A, Glaser S. et al. Ventilatory efficiency and exercise tolerance in 101 healthy volunteers. Eur J Appl Physiol 1998;77:421-6.

6. Cooper DM, Weiler-Ravell D, Whipp BJ, Wasserman K. Aerobic parameters of exercise as a function of body size during growth in children. J Appl Physiol 1984;56:628-34.

7. Marinov B, Kostianev S, Turnovska T. Ventilatory efficiency and rate of perceived exertion in obese and non-obese children performing standardized exercise. Clin Physiol \& Func Im 2002;22:254-60.

8. Nagano Y, Baba R, Kuraishi K, Yasuda, T, Ikoma M, Nishibata K, et al. Ventilatory control during exercise in normal children. Pediatr Res 1998;43:704-7.

9. Wasserman K, McIlroy MB. Detecting the threshold of anaerobic metabolism in cardiac patients during exercise. Am J Cardiol 1964;14:844-52.

10. Wasserman K, Whipp BJ, Royal SN, Beaver WL. Anaerobic threshold and respiratory gas exchange during exercise. J Appl Physiol 1973;35(2):236-3.

11. Davis JA. Anaerobic threshold: review of the concept and directions for future research. Med Sci Sports Exer 1985;17:6-18.

12. Wasserman K, Beaver WL, Whipp BJ. Gas exchange theory and the lactic acidosis (anaerobic) threshold. Circulation 1990;81(suppl II):II-14-Il-30.

13. Anderson CS, Mahon AD. The relationship between ventilatory and lactate threshold in boys and men. Res Sports Med 2007;15:189-200.

14. Myers J, Ashley E. Dangerous curves. A perspective on exercise, lactate, and the anaerobic threshold. Chest 1997;111:787-95.

15. Beaver WL, Wasserman K, Whipp BJ. A new method for detecting anaerobic threshold by gas exchange. J Appl Physiol 1986;60:2020-7.

16. Davis JA, Frank MH, Whipp BJ, Wasserman K. Anaerobic threshold alterations caused by endurance training in middle-age men. J Appl Physiol 1979;46:1039-46.

17. Hebestreit H, Staschen B, Hebestreit A. Ventilatory threshold: a useful method to determine aerobic fitness in children? Med Sci Sports Exerc 2000;32(11):1964-9.

18. Meyer K, Hajric R, Westbrook S, Samek L, Lehmann M, Schwaibold M, et al. Ventilatory and lactate threshold determinations in healthy normals and cardiac patients: methodological problems. Eur J Appl Physiol 1996;72:387-93.

19. Mahon AD, Vaccaro P. Ventilatory threshold and VO2 max changes in children following endurance training. Med Sci Sports Exer 1989;21:425-31.

20. Reybrouck T, Weymans M, Stijns H, Knops J, Van der Hauwaert L. Ventilatory anaerobic threshold in healthy children. Eur J Appl Physiol 1985;54:278-84.

21. Rowland TW, Green GM. Physiological responses to treadmill exercise in females: adult-child differences. Med Sci Sports Exerc 1988;20:474-8.

22. Vago P, Mercier J, Ramonatxo M, Prefaut C. Is ventilatory anaerobic threshold a good index of endurance capacity? Int J Sports Med 1987;8:190-5.

23. Paterson DH, McLellan TM, Stella RS, Cunningham DA. Longitudinal study of ventilation threshold and maximal O2 uptake in athletic boys. J Appl Physiol 1987;62:2051-7.

24. Meyer T, Lucia A, Earnest CP, Kinderman W. A conceptual framework for performance diagnosis and training prescription from submaximal gas exchange parameters-theory and application. Int J Sports Med 2005;26(Suppl 1):S38-S48.

25. Shinohara T, Suzuki N, Oba M, Kawasumi M, Kimizuka M, Mita K. Effect of exercise at the AT point for children with cerebral palsy. Bulletin of the NYU Hospital for Joint Diseases 2002-2003;61(1 \& 2):63-7.

26. Rhodes J, Curran T, Camil L, Rabideau N, Fulton D, Gauthier N, et al. Impact of cardiac rehabilitation on the exercise function of children with serious congenital heart disease. Pediatrics 2005;116:1339-45.

27. Wasserman $\mathrm{K}$. The anaerobic threshold measurement to evaluate exercise performance. Am Rev Respir Dis 1984;129(2):S35-S40.

28. Wasserman $\mathrm{K}$. The anaerobic threshold measurement in exercise testing. Clin Chest Med 1984;5(1):77-88. 
29. Wasserman K, Koike A. Is the anaerobic threshold truly anaerobic? Chest 1992;101:211S-218S.

30. Gaskill SE, Ruby BC, Walker AJ, Sanchez OA, Serfass RC, Leon AS. Validity and reliability of combining three methods to determine ventilatory threshold. Med Sci Sports Exerc 2001;33:1841-8.

31. Dickstein K, Barvik S, Aarsland T, Snapinn S, Karlsson J. A comparison of methodologies in detection of the anaerobic threshold. Circulation 1990;81:38-46.

32. Moalla W, Dupont G, Berthoin S, Ahmaidi S. Respiratory muscle deoxygenation and ventilatory threshold assessments using near infrared spectroscopy in children. Int J Sports Med 2005;26:576-82.

33. Fernandez A, Cintra I, Sawaya A, Fisberg M, Silva A. Respostas metabólicas e cardiorrespiratórias ao exercício máximo e submáximo em meninas eutróficas e com desnutrição pregressa. Rev Ass Med Brasil 2000; 46(4):312-9.

34. Zeballos RJ, Weisman IM. Behind the scenes of cardiopulmonary exercise testing. In: Weisman IM, Zeballos RJ. Clinics in chest medicine: clinical exercise testing. W.B. Saunders Company 1994;15(2):193-214.

35. Sritippayawan S, Harnruthakorn C, Deerojanawong J, Samransamruajkit R, Prapphal N. Optimal level of physical activity in children with chronic lung diseases. Acta Pædiatrica 2008;97:1582-7.

36. Solberg G, Robstad B, Skjønsberg OH, Borchsenius F. Respiratory gas exchange indices for estimating the anaerobic threshold. J Sports Sci Med 2005;4:29-36.

37. Armstrong N, Fawkner SG. Non-invasive methods in paediatric exercise physiology. Appl Physiol Nutr Metab 2008;33:402-10.

38. Wasserman K, Hansen JE, Sue DY, Casaburi R, Whipp BJ. Prova de esforço: princípio e interpretação. Rio de Janeiro: Revinter; 2005.
Endereço para correspondência

Anselmo José Perez

Rua Antiocho Carneiro de Mendonça

170/807 - Jardim Camburi

CEP: 29092-130 - Vitória, ES. Brasil

E-mail: anselmo@cefd.ufes.br 